\title{
Variations in the prevalence of childhood asthma and wheeze in MeDALL cohorts in Europe
}

\section{Eleonora P. Uphoff ${ }^{1,2}$, Philippa K. Bird ${ }^{1}$, Joseph Maria Antó3, Mikel Basterrechea ${ }^{4,5,6}$, Andrea von Berg ${ }^{7}$, Anna Bergström ${ }^{8}$, Jean Bousquet ${ }^{9}$, Leda Chatzi $^{10}$, Maria Pia Fantini ${ }^{11}$, Amparo Ferrero ${ }^{4,12}$, Ulrike Gehring ${ }^{13}$, Davide Gori ${ }^{11}$, Joachim Heinrich ${ }^{14}$, Thomas Keil ${ }^{15}$, Inger Kull ${ }^{8}$, Susanne Lau ${ }^{16}$, Dieter Maier ${ }^{17}$, Isabelle Momas ${ }^{18}$, Silvia Narduzzi ${ }^{19}$, Daniela Porta ${ }^{19}$, Fanny Ranciere ${ }^{18}$, Theano Roumeliotaki ${ }^{10}$, Tamara Schikowski ${ }^{20}$, Henriette A. Smit ${ }^{21}$, Marie Stand1 ${ }^{14}$, Jordi Sunyer ${ }^{3}$ and John Wright ${ }^{1}$}

Affiliations: ${ }^{1}$ Bradford Institute for Health Research, Bradford, UK. ${ }^{2}$ Dept of Health Sciences, University of York, York, UK. ${ }^{3}$ Insituto de Salud Global Barcelona, Barcelona, Spain. ${ }^{4}$ CIBER Epidemiología y Salud Pública, Madrid, Spain. ${ }^{5}$ Subdirectorate of Public Health of Gipuzkoa, Basque Government, Dept of Health, Gipuzkoa, Spain. ${ }^{6}$ Health Research Institute BIODONOSTIA, Gipuzkoa, Spain. ${ }^{7}$ Dept of Pediatrics, Marien Hospital Wesel, Wesel, Germany. ${ }^{8}$ Institute of Environmental Medicine, Karolinska Institute, Stockholm, Sweden. ${ }^{9}$ Centre Hôpital Universitaire de Montpellier, Montpellier, France. ${ }^{10}$ Dept of Social Medicine, University of Crete, Heraklion, Greece. ${ }^{11}$ Dipartimento di Scienze Biomediche e Neuromotorie, Universita degli Studi di Bologna, Bologna, Italy. ${ }^{12}$ FISABIO, Universitat de València-Universtitat Jaume I, Joint Research Unit, Valencia, Spain. ${ }^{13}$ Institute for Risk Assessment Sciences, Utrecht University, Utrecht, The Netherlands. ${ }^{14}$ Institute of Epidemiology, Helmholtz Zentrum München, Munich, Germany. ${ }^{15}$ Social Medicine, Epidemiology and Health Economics, Charité Medical University, Berlin, Germany. ${ }^{16}$ Pediatric Pneumology and Immunology, Charité Medical University, Berlin, Germany. ${ }^{17}$ Biomax Informatics AG, Planegg, Germany. ${ }^{18}$ Université Paris Descartes, Paris, France. ${ }^{19}$ Dipartimento di Epidemiologia del SSR del Lazio, Rome, Italy. ${ }^{20}$ Leibniz-Institut für umweltmedizinische Forschung, Heinrich Heine Universitat, Düsseldorf, Germany. ${ }^{21}$ Julius Center for Health Sciences and Primary Care, Public Health, Health Technology Assessment, Medical Humanities, University Medical Center Utrecht, Utrecht, The Netherlands.

Correspondence: Eleonora P. Uphoff, Dept of Health Sciences, University of York, Seebohm Rowntree Building, York, Y010 5DD, UK. E-mail: noortje.uphoffayork.ac.uk

ABSTRACT While there is evidence for variations in prevalence rates of childhood wheeze and asthma between countries, longitudinal, individual-level data are needed to understand these differences. The aim of this study was to examine variations in prevalence rates of childhood asthma, wheeze and wheeze with asthma in Europe.

We analysed datasets from 10 MeDALL (Mechanisms of the Development of ALLergy) cohorts in eight countries, representing 26663 children, to calculate prevalence rates of wheeze and asthma by child age and wheeze with asthma at age 4 years. Harmonised variables included outcomes parent-reported wheeze and parent-reported doctor-diagnosed asthma, and covariates maternal education, parental smoking, pets, parental asthma, doctor-diagnosed allergic rhinitis, doctor-diagnosed eczema and wheeze severity.

At age 4 years, asthma prevalence varied from $1.72 \%$ in Germany to $13.48 \%$ in England and the prevalence of wheeze varied from $9.82 \%$ in Greece to $55.37 \%$ in Spain. Adjusted estimates of the proportion of 4-year-old children with wheeze diagnosed with asthma remained highest in England (38.14\%, 95\% CI 31.38-44.90\%) and lowest in Spain (15.94\%, 95\% CI 6.16-25.71\%).

The large differences in prevalence rates of asthma, wheeze and wheeze with asthma at age 4 years between European cohorts may indicate that childhood asthma is more readily diagnosed in some countries while going unrecognised elsewhere.

@ERSpublications

Large variations in childhood wheeze across Europe do not match large variations in diagnosed childhood asthma http://ow.ly/eJQk30aPInr

Cite this article as: Uphoff EP, Bird PK, Antó JM, et al. Variations in the prevalence of childhood asthma and wheeze in MeDALL cohorts in Europe. ERJ Open Res 2017; 3: 00150-2016 [https:// doi.org/10.1183/23120541.00150-2016].

Copyright $\odot$ ERS 2017. This article is open access and distributed under the terms of the Creative Commons Attribution Non-Commercial Licence 4.0. 


\section{Introduction}

\section{Global burden of disease}

In the International Study of Asthma and Allergies in Childhood (ISAAC), the global prevalence of wheeze was estimated to be $11.6 \%$ for $6-7$-year-old children and $13.7 \%$ for 13 -14-year-old children for the period around 2001-2003 [1]. Wheeze is particularly prevalent in English-speaking and Western countries [2]. Far less data are available on international prevalence rates of doctor-diagnosed asthma in children. The ISAAC study estimated the global prevalence of parent-reported doctor-diagnosed asthma among 6-7-year-old children to be $10.8 \%$, with lower rates in Northern and Eastern Europe (4.5\%), and the highest rates in North America (20.0\%) and Oceania (29.2\%). This pattern was repeated in 13-14-year-old children, with figures suggesting a small increase with age of $\sim 2 \%$ in most countries except for Western Europe (9.1\% among 6-7-year-olds to $16.3 \%$ among $13-14$-year-olds) [1].

\section{Wheeze versus doctor-diagnosed asthma}

Self-reported wheeze is often used as an indicator of asthma [1]. While wheeze and asthma frequently go hand-in-hand, wheeze is not a sufficient, nor a necessary symptom, for the diagnosis of asthma. Partly due to the highly variable expression of asthma, there is no uniform set of diagnostic criteria [3]. SPYCHER et al. [4] showed that only $2.9 \%$ of all children in the general population present with the combination of wheeze, atopy and bronchial hyperresponsiveness described as typical for asthma. In another study, 25.8\% of young adults with asthma never experienced childhood wheeze and of the children with wheeze, $51.8 \%$ did not develop asthma [5]. If differences between prevalence rates of wheeze and asthma vary internationally, using wheeze as a proxy for asthma is problematic.

\section{Gaps in the evidence}

Despite evidence of varying prevalence rates, no international comparisons of wheeze in combination with doctor-diagnosed asthma by child age are available. The ISAAC survey only measures self-reported wheeze and asthma in two age groups consisting of different samples [1]. Studying these variations between countries could lead to a greater understanding of modifiable factors involved, e.g. relating to the environment or the diagnostic process. The MeDALL (Mechanisms of the Development of ALLergy) partnership provides individual-level, longitudinal data with harmonised measures of wheeze and doctor-diagnosed asthma across cohorts [6]. We aimed to examine variations in the prevalence of childhood asthma, wheeze and wheeze with asthma by child age in Europe.

\section{Methods}

We analysed cross-sectional differences in childhood asthma and wheeze by child age and wheeze with asthma at age 4 years in datasets from European cohorts with harmonised variables on asthma and wheeze. Data were requested from 13 MeDALL cohort studies [6]. Table 1 provides an overview of the 10 included cohorts, representing eight countries and a total sample of 26663 children. More information about the cohorts can be found in the ENRIECO database (www.enrieco.dk).

\section{Measures}

The MeDALL partnership has developed harmonised variables, including harmonised definitions of asthma and wheeze [7]. "Ever asthma" is defined by the question "Has your child ever been diagnosed by a doctor as having asthma?" and "ever wheeze" is captured by the question "Has your child ever had wheezing or whistling in the chest?" For BAMSE, LISAplus and GINIplus cohorts, only wheeze and doctor diagnosis of asthma in the past 12 months were available in the follow-ups analysed, and composite measures based on annual reports of wheeze and/or asthma were created where possible (supplementary table S1). A third outcome measure was constructed to capture the proportion of children whose parents reported wheeze at least once from birth until age 4 years and who were reported to be diagnosed with asthma by age 4 years.

Key covariates included maternal education (low/medium/high, cohort specific), parental smoking up to child age 4 years, pets in the household up to child age 4 years (dog and/or cat and/or other pets, cohort specific), parental ever asthma, ever doctor-diagnosed allergic rhinitis and ever doctor-diagnosed eczema.

This article has supplementary material available from openres.ersjournals.com

Received: Dec 122016 | Accepted after revision: March 082017

Support statement: The MeDALL collaboration is funded by European Union Seventh Framework Programme grant agreement number 261357. Funding information for this article has been deposited with the Crossref Funder Registry.

Conflict of interest: Disclosures can be found alongside this article at openres.ersjournals.com 
TABLE 1 Overview of included MeDALL birth cohorts

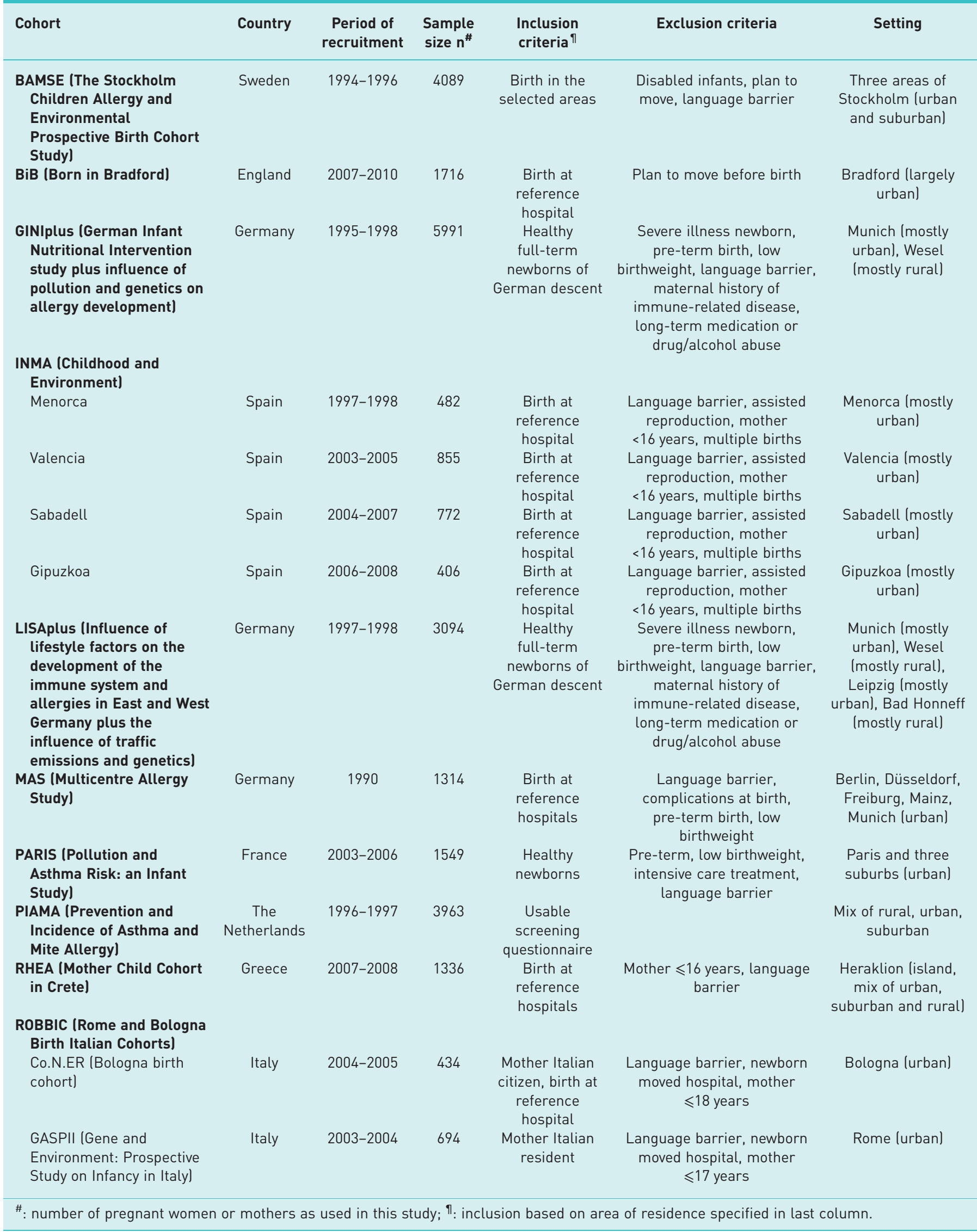


Severity of wheeze was considered a potential explanatory variable in the relationship between cohort and percentage of children with wheeze diagnosed with asthma. However, as it was only available in five cohorts, it was used in additional analyses only. The measure was constructed from the question "How many attacks of wheezing has your child had in the last 12 months?" and coded low (0 times), medium or high severity (coding varied by cohort).

\section{Statistical analysis}

Prevalence rates of wheeze and asthma were calculated up to age 8 years, because four cohorts had no data from later time points (ROBBIC, RHEA, BiB and PARIS) and for many other cohorts a high loss to follow-up affected the reliability of the data (supplementary table S1).

Data for subcohorts in INMA and ROBBIC were pooled, although data availability differed across subcohorts for different time points (table 1). Cross-sectional logistic regression analyses were performed separately for outcomes asthma at age 4 years, wheeze at age 4 years and wheeze with diagnosed asthma at age 4 years. Unadjusted prevalence rates are presented by child age for all cohorts, and unadjusted and adjusted prevalence rates are presented for all cohorts at age 4 years, as this was the follow-up age with most data available across cohorts. Prevalence rates at age 4 years could not be calculated for the ROBBIC, MAS, GINIplus and LISAplus cohorts, because these studies did not measure the relevant outcomes at age 4 years.

\section{Results}

Characteristics of the cohort participants by cohort are described in supplementary table S2. Unadjusted prevalence rates and output from regression analyses are reported in supplementary table S3.

\section{Prevalence rates of asthma and wheeze by child age}

In all cohorts with longitudinal data, figure 1 suggests a trend for prevalence rates of asthma to increase with child age. Differences between prevalence rates at the first and last time point are statistically significant for each cohort (supplementary table S3). However, there were large differences between cohorts across the entire age range. Reported ever wheeze did not consistently increase with child age (figure 2).

\section{Ever asthma at age 4 years}

At age 4 years, for which eight cohorts provided data, unadjusted prevalence rates of ever asthma varied from $1.72 \%$ (95\% CI $1.22-2.35 \%$ ) in Germany (LISAplus) to $13.48 \%$ (95\% CI $11.88-15.20 \%$ ) in England (BiB) (figure 3).

Asthma at age 4 years was not associated with the presence of pets in the home (OR 1.02, 95\% CI $0.88-1.18$ ), but was associated with medium (OR 0.84, 95\% CI 0.70-1.00) and high (OR 0.74, 95\% CI $0.62-0.88$ ) versus low maternal education, parental smoking (OR 1.31, 95\% CI 1.10-1.57), parental asthma (OR 2.38, 95\% CI 2.07-2.73), doctor-diagnosed allergic rhinitis (OR 4.12, 95\% CI 3.23-5.25) and doctor-diagnosed eczema (OR 2.21, 95\% CI 1.93-2.53).

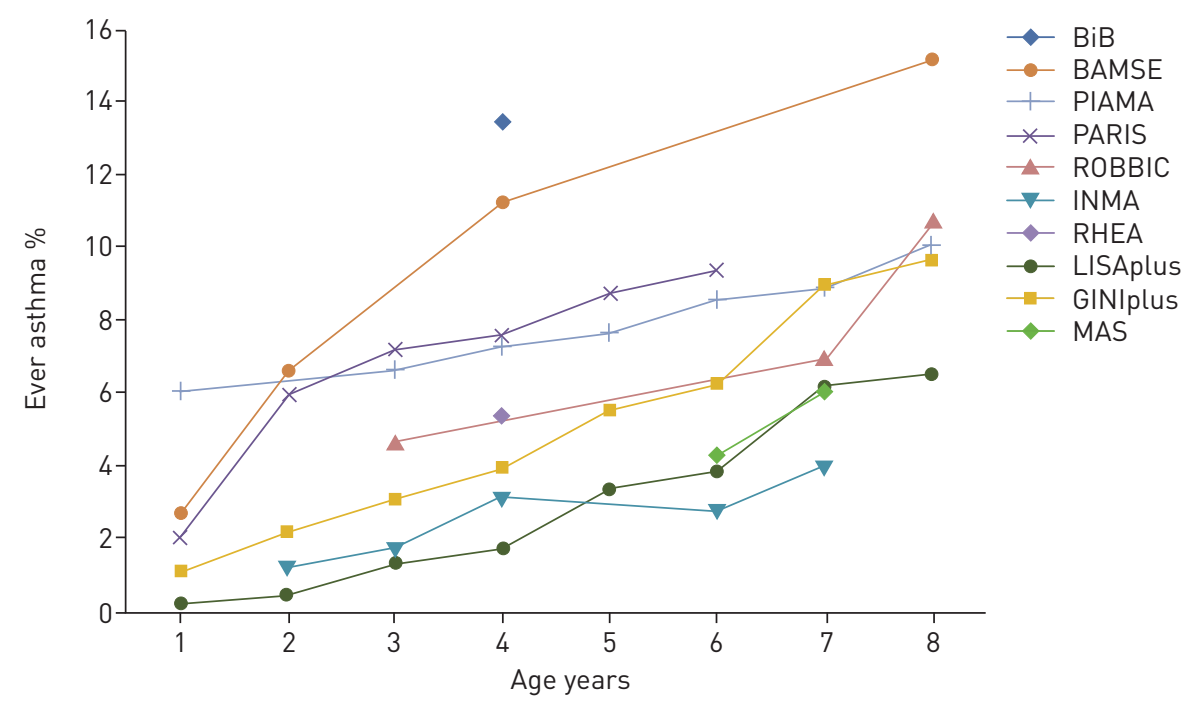

FIGURE 1 Prevalence rates of ever asthma by cohort (table 1) and child age. 


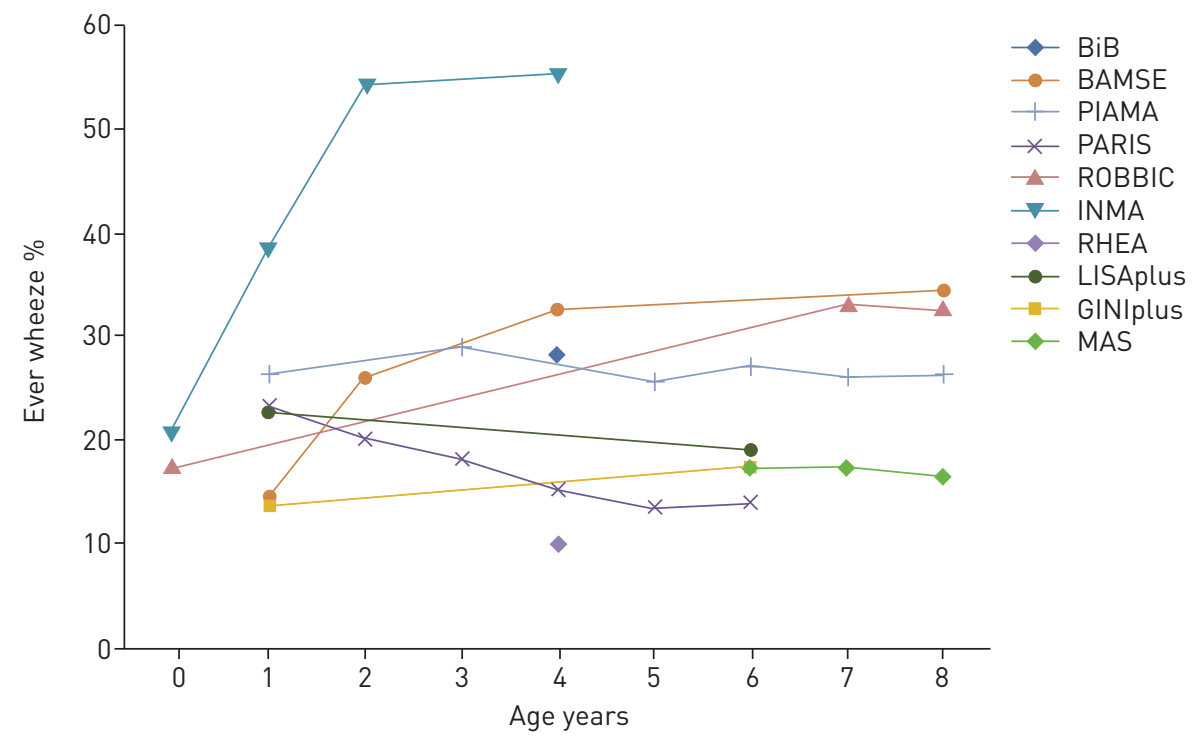

FIGURE 2 Prevalence rates of ever wheeze by cohort (table 1) and child age.

After adjusting for these covariates, prevalence rates shown in figure 3 indicate that large and consistent differences in diagnosed asthma at age 4 years remain. Again, the highest prevalence rates of asthma are found in England (BiB), Sweden (BAMSE) and France (PARIS), while Germany (GINIplus and LISAplus) and Spain (INMA) have the lowest rates.

Ever wheeze at age 4 years

There was a wide variation in prevalence rates of ever wheeze at age 4 years. However, cohorts with the highest average prevalence of asthma at age 4 years (figure 3 ) do not have the highest average prevalence of wheeze at age 4 years (figure 4). Unadjusted prevalence rates of wheeze at age 4 years for six cohorts with available data varied from 9.82\% (95\% CI 7.95-11.96\%) in Greece (RHEA) to 55.37\% (95\% CI $52.21-58.51 \%$ ) in Spain (INMA).

Wheeze at age 4 years was not associated with maternal education (OR 1.03, 95\% CI 0.93-1.15 and OR 1.01, 95\% CI $0.91-1.12$ for low and medium versus high education, respectively) nor with pets in the home (OR 1.05, 95\% CI 0.97-1.14), but was associated with parental smoking (OR 1.42, 95\% CI

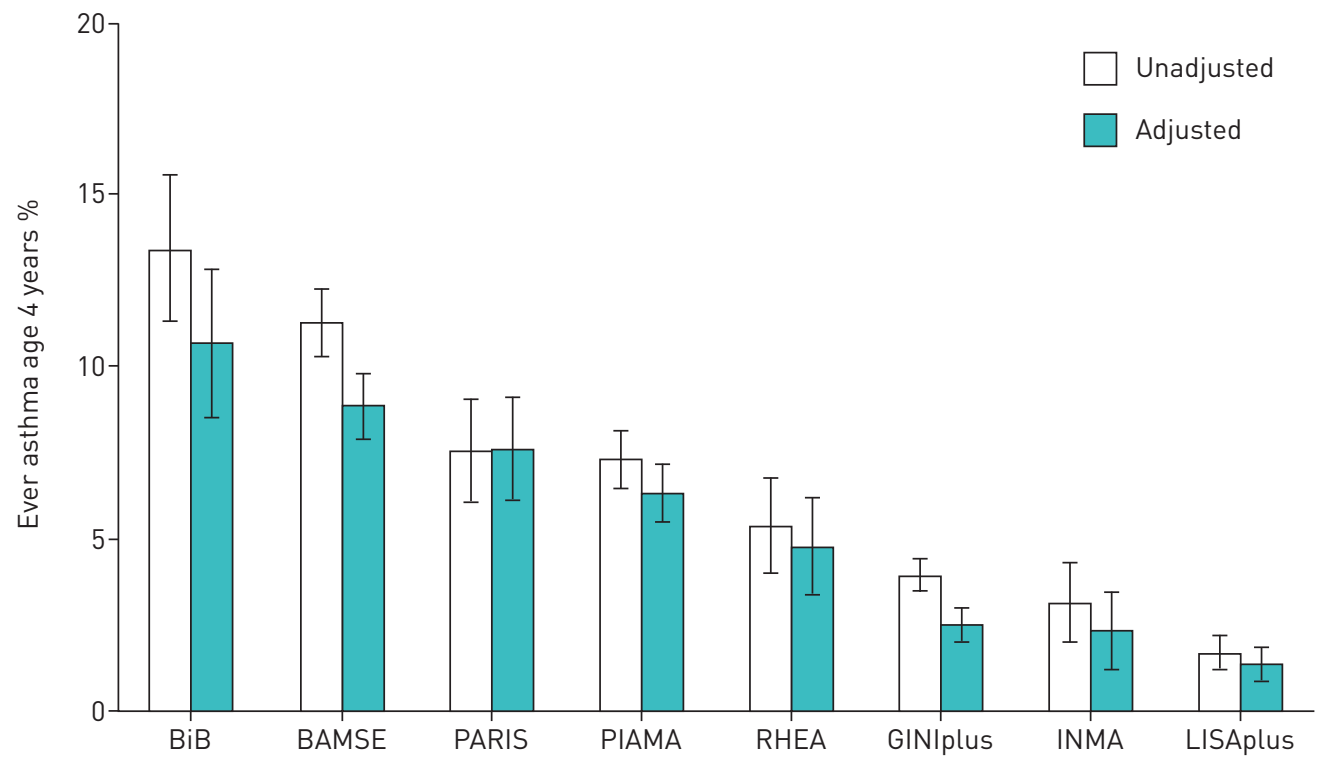

FIGURE 3 Unadjusted and adjusted prevalence rates of ever asthma at age 4 years by cohort (table 1). 
FIGURE 4 Unadjusted and adjusted prevalence rates of ever wheeze at age 4 years by cohort (table 1).

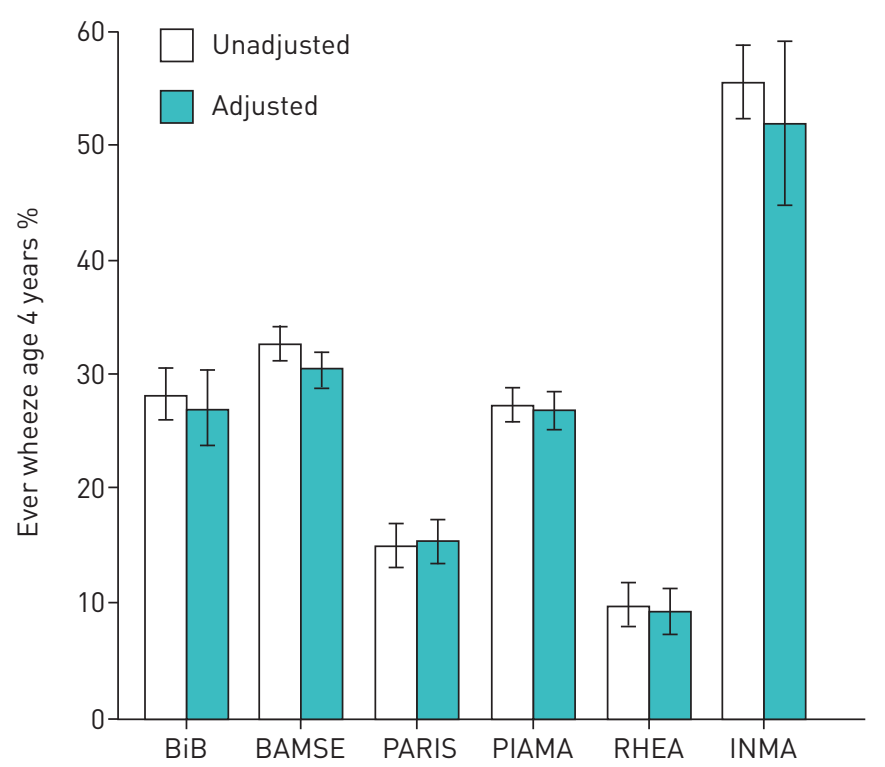

1.28-1.58), parental asthma (OR 1.71, 95\% CI 1.57-1.87), doctor-diagnosed allergic rhinitis (OR 2.24, 95\% CI 1.84-2.71) and doctor-diagnosed eczema (OR 1.52, 95\% CI 1.40-1.65). Adjusted prevalence rates of wheeze remain highest in Spain (INMA), and lowest in France (PARIS) and Greece (RHEA) (figure 4).

\section{Children with ever wheeze diagnosed with asthma by age 4 years}

The proportion of children with ever wheeze who were diagnosed with asthma by age 4 years ranged from $15.94 \%$ (95\% CI $8.24-26.74 \%$ ) in Spain (INMA) to $39.53 \%$ (95\% CI 35.07-44.12\%) in England (BiB) (figure 5).

Wheeze with diagnosed asthma compared with wheeze without diagnosed asthma at age 4 years was not associated with low- versus medium-level maternal education (OR 0.83, 95\% CI 0.65-1.05), but children with wheeze were less likely to be diagnosed with asthma if their mother had a low versus high level of education (OR 0.71, 95\% CI 0.56-0.90). There was no association with parental smoking (OR 1.05, 95\% CI $0.83-1.34$ ) or pets (OR 1.01,95\% CI 0.82-1.24), but wheeze with asthma was more likely in children of parents with asthma (OR 1.85, 95\% CI 1.52-2.24), children diagnosed with allergic rhinitis (OR 2.81, 95\% CI 1.86-4.24) and children diagnosed with eczema (OR 1.68, 95\% CI 1.40-2.03).

The probability of children with wheeze being diagnosed with asthma by age 4 years was also increased for medium versus low (OR 1.90, 95\% CI 1.52-2.39) and high versus low wheeze severity (OR 5.65, 95\% CI



FIGURE 5 Prevalence rates of wheeze with diagnosed asthma at age 4 years by cohort (table 1). 
4.24-7.54). However, adding wheeze severity to the model did not reduce between-cohort differences (figure 5). Estimates for children with wheeze diagnosed with asthma in the fully adjusted model were higher for England $(\mathrm{BiB})(49.17 \%$, 95\% CI 39.17-59.18\%) and France (PARIS) $(52.07 \%$, 95\% CI 40.79-63.35\%) than for Sweden (BAMSE) $(27.06 \%$, 95\% CI 24.07-30.05\%), the Netherlands (PIAMA) (22.49\%, 95\% CI 19.37-25.61\%) and Spain (INMA) (15.31\%, 95\% CI 5.58-25.04\%).

\section{Discussion}

Prevalence rates of childhood asthma and wheeze vary by country, with a seven-fold variation in asthma across cohorts at age 4 years and persisting differences at older ages. Whereas the prevalence of asthma generally increases with age, the prevalence of wheeze does not. The highest prevalence rates of asthma were found among cohorts in England, Sweden and France, and the lowest in Germany and Spain. The highest rates of wheeze were found in Spain, Italy and Sweden, and the lowest in Germany, France and Greece. Although we showed strong associations with covariates parental smoking, parental asthma, allergic rhinitis and eczema, these factors did not explain between-cohort differences in prevalence rates.

Discrepancies between prevalence rates of asthma and wheeze were confirmed by differences between cohorts in the proportion of children with wheeze diagnosed with asthma by age 4 years. Children with wheeze were more likely to be diagnosed with asthma if their mother had a higher level of education, if the child was diagnosed with allergic rhinitis or eczema and if wheeze was more severe. However, even after adjusting for these covariates, children with wheeze were more likely to be diagnosed with asthma in England and France than in Sweden, the Netherlands and Spain. We consider a few explanations for these variations.

\section{Explaining variations between European cohorts}

To put our findings in perspective, we compared our results with estimates from international surveys (supplementary appendix S1 and supplementary figure S1). Although no data on doctor-diagnosed childhood asthma were available, findings from two surveys among adults suggest findings in line with ours, with the highest prevalence rates of asthma in the UK, Sweden, France and the Netherlands, and consistently lower prevalence rates in Germany, Spain, Greece and Italy [8, 9]. Our estimates of wheeze in Spain and Italy are higher than previously found, but these cohorts are not representative of the general population (supplementary table S4).

Variations may be attributable to international differences in the diagnosis of childhood asthma or guideline adherence. The PRACTALL (Practical Allergy) consensus recommendations provide guidance for diagnosis and treatment of childhood asthma in Europe and North America, and state that suspected asthma can only be diagnosed through follow-up and a trial of treatment [10]. Current Dutch guidelines are even more cautious and discourage the diagnosis of asthma for children under the age of 6 years [11]. UK guidelines recommend a trial of treatment or watchful waiting for those under the age of 6 years, but state that more frequent and/or severe symptoms add certainty to the asthma diagnosis [12]. Swedish guidelines prioritise lung function and allergy tests even for those under the age of 4 years; in France, radiographs are recommended as standard practice [13, 14]. Furthermore, there are indications of underdiagnosis of childhood asthma, e.g. in Italy [15], and evidence of overdiagnosis in the Netherlands [16]. One study found that asthmatic complaints which often led to an asthma diagnosis for children in the Netherlands were frequently diagnosed and treated as bronchitis in Germany [17]. These differences in healthcare may contribute to some of the variation in prevalence rates of asthma and wheeze with diagnosed asthma found in our study.

Alternatively, limited access to healthcare and differences in the translation and interpretation of "wheeze" might play a role in various countries. The strength of the relationship between wheeze and asthma may also vary between countries. A recent study comparing childhood mortality rates found that the mortality rate for paediatric respiratory disorders in England was 6.6 times that of Sweden, despite the prevalence of childhood asthma being high in both countries [18].

\section{Implications for research}

Future research should aim to explain variations in childhood wheeze and diagnosed asthma in longitudinal analyses, e.g. in age-period-cohort analyses. This could determine whether rates of asthma in some countries are rising over time, rather than with age alone. We also suggest that, in addition to environmental factors such as air pollution and climate, international variations in diagnoses, access to healthcare and quality of care deserve consideration.

A second implication for research concerns the findings that, while the prevalence of childhood asthma increased with child age, wheeze did not. Wheeze is not a reliable indicator of asthma at an early age when many young children are yet to be diagnosed. The levelling in the prevalence of ever wheeze with 
increasing child age suggests that these estimates are affected by recall bias. Parents may not remember transient wheeze suffered by their children at an early age if it does not progress to asthma. A previous version of the UK guidelines warned that "Parents often use 'wheezing' as a non-specific label ..." [12]. Using parent-reported wheeze as an indicator of asthma for young children therefore leads to overestimates of the prevalence of childhood asthma.

\section{Implications for practice}

Consensus recommendations have not yet led to an internationally uniform diagnosis of childhood asthma [10]. Our results suggest that the likelihood of children with asthma being diagnosed, and subsequently the quality of care, may vary internationally. We have shown that in many countries children are diagnosed with asthma at age 4 years or earlier, while it is doubtful whether a diagnosis of childhood asthma can be made with certainty at such a young age. It will take the joint effort of healthcare professionals and researchers to ensure the diagnosis of childhood asthma is uniform and accurate.

\section{Limitations}

As shown in table 1, cohorts used different inclusion and exclusion criteria, and participants were recruited from centres not representative of the general population. Limited generalisability is particularly problematic for cohorts using exclusion criteria related to the risk of asthma, such as mothers who do not speak the language of the country and infants with complications at birth [19-21]. This, in addition to variations in time periods of data collection and the limited availability of harmonised covariates at different follow-ups, means that comparisons of prevalence rates between cohorts and within cohorts over time should be made with caution. For example, composed outcome variables created for some cohorts were based on data from multiple time points, leading to overestimation. Despite the availability of data at multiple time points, our analysis was cross-sectional and measured asthma and wheeze "ever" rather than "in the last 12 months" due to data limitations. However, we believe our conclusions are strengthened by taking into account key covariates, by the fact that differences between cohorts persist at different child ages and that these generally correspond with results from other surveys.

\section{Conclusions}

There are differences in prevalence rates of childhood asthma and wheeze across Europe, which vary by child age. The proportion of children with wheeze who were diagnosed with asthma was higher among cohorts in England, Sweden and France than in the Netherlands and Spain, even when the higher proportion of severe wheeze in England and France was adjusted for. This may partly indicate that asthma is more readily diagnosed in some countries, while going unrecognised in others. Given the burden on patients and healthcare associated with overdiagnosis as well as underdiagnosis, the discussion on diagnostic criteria for asthma should be an international one.

\section{Acknowledgements}

This study would not have been possible without the commitment of the cohort participants and our colleagues working with the various European birth cohorts.

\section{References}

1 Pearce N, Aït-Khaled N, Beasley R, et al. Worldwide trends in the prevalence of asthma symptoms: phase III of the International Study of Asthma and Allergies in Childhood (ISAAC). Thorax 2007; 62: 758-766.

2 Pearce N, Sunyer J, Cheng S, et al. Comparison of asthma prevalence in the ISAAC and the ECRHS. ISAAC Steering Committee and the European Community Respiratory Health Survey. International Study of Asthma and Allergies in Childhood. Eur Respir J 2000; 16: 420-426.

3 Scottish Intercollegiate Guidelines Network/British Thoracic Society. British Guideline on the Management of Asthma (2014 revision; SIGN 141). Edinburgh, SIGN/BTS, 2014.

4 Spycher BD, Silverman M, Kuehni CE. Phenotypes of childhood asthma: are they real? Clin Exp Allergy 2010; 40: $1130-1141$.

5 Stern DA, Morgan WJ, Halonen M, et al. Wheezing and bronchial hyper-responsiveness in early childhood as predictors of newly diagnosed asthma in early adulthood: a longitudinal birth-cohort study. Lancet 2008; 372 $1058-1064$.

6 Bousquet J, Anto J, Auffray C, et al. MeDALL (Mechanisms of the Development of ALLergy): an integrated approach from phenotypes to systems medicine. Allergy 2011; 66: 596-604.

7 Hohmann C, Pinart M, Tischer C, et al. The development of the MeDALL Core Questionnaires for a harmonized follow-up assessment of eleven European birth cohorts on asthma and allergies. Int Arch Allergy Immunol 2014; 163: 215-224.

8 To T, Stanojevic S, Moores G, et al. Global asthma prevalence in adults: findings from the cross-sectional world health survey. BMC Public Health 2012; 12: 204-204.

9 European Community Respiratory Health Survey. Variations in the prevalence of respiratory symptoms, self-reported asthma attacks, and use of asthma medication in the European Community Respiratory Health Survey (ECRHS). Eur Respir J 1996; 9: 687-695. 
10 Bacharier LB, Boner A, Carlsen $\mathrm{KH}$, et al. Diagnosis and treatment of asthma in childhood: a PRACTALL consensus report. Allergy 2008; 63: 5-34.

11 Bindels P, Van de Griendt E, Grol M, et al. NHG-Standaard Asthma by Kinderen (derde herziening). [NHG-Standard Asthma in Children (third review).] Huisarts Wet 2014; 57: 70-80.

12 Scottish Intercollegiate Guidelines Network/British Thoracic Society. British Guideline on the Management of Asthma, 2009 revision (SIGN 101). Edinburgh, SIGN/BTS, 2009.

13 Jonsson M, Egmar AC, Kiessling A, et al. Adherence to national guidelines for children with asthma at primary health centres in Sweden: potential for improvement. Prim Care Respir J 2012; 21: 276-282.

14 Haute Autorité de Santé. Asthme de l'enfant de moins de 36 mois: diagnostic, prise en charge et traitement en dehors des épisodes aigus. [Asthma of children under 36 months: diagnosis, management and treatment outside acute episodes.] Paris, HAS, 2009.

15 Magnoni MS, Caminati M, Senna G, et al. Asthma under/misdiagnosis in primary care setting: an observational community-based study in Italy. Clin Mol Allergy 2015; 13: 26.

16 Looijmans-van den Akker I, van Luijn K, Verheij T. Overdiagnosis of asthma in children in primary care: a retrospective analysis. Br J Gen Pract 2016; 66: e152-e157.

17 Mommers M, Swaen GM, Weishoff-Houben M, et al. Differences in asthma diagnosis and medication use in children living in Germany and the Netherlands. Prim Care Respir J 2005; 14: 31-37.

18 Tambe P, Sammons HM, Choonara I. Why do young children die in the UK? A comparison with Sweden. Arch Dis Child 2015; 100, 928-931.

19 Murphy V, Namazy J, Powell $\mathrm{H}$, et al. A meta-analysis of adverse perinatal outcomes in women with asthma. BJOG 2011; 118: 1314-1323.

20 Jaakkola JJ, Ahmed P, Ieromnimon A, et al. Preterm delivery and asthma: a systematic review and meta-analysis. J Allergy Clin Immunol 2006; 118: 823-830.

21 Cabieses B, Uphoff E, Pinart M, et al. A systematic review on the development of asthma and allergic diseases in relation to international immigration: the leading role of the environment confirmed. PLoS One 2014; 9: e105347. 